\title{
Contactless online characterization of large-area conductive thin films by thermography and induction
}

\author{
KARI REMES, ${ }^{1,}{ }^{*}$ ANTTI JÄRVENPÄÄ, ${ }^{2}$ TAPIO FABRITIUS ${ }^{1}$ \\ ${ }^{1}$ Optoelectronics and Measurement Techniques, Faculty of Information Technology and Electrical Engineering, University of Oulu, Erkki Koiso- \\ Kanttilankatu 3, 90570 Oulu, Finland \\ 2 Future Manufacturing Technologies, Kerttu Saalasti Institute, University of Oulu, Pajatie 5, 85500 Nivala, Finland \\ *Corresponding author: kari.remes@oulu.fi
}

Received XX Month XXXX; revised XX Month, XXXX; accepted XX Month XXXX; posted XX Month XXXX (Doc. ID XXXXX); published XX Month XXXX

Testing and characterization techniques intended for traditional electronics production are rarely compatible with modern large-area, thin film electronics manufacturing processes such as roll-to-roll fabrication. Online quality monitoring of conductive thin films is necessary for upscaling and maintaining high-yield production. Thermography has already shown its usefulness in this kind of applications but has suffered the lack of proper non-contact electrical heating. Now, a fully contactless quality inspection technique based on thermal imaging and induction heating is implemented and evaluated. This approach is capable to find out defected areas and to estimate conductivity degradation online with full coverage over conductive thin films. (C) 2019 Optical Society of America

http://dx.doi.org/10.1364/OL.99.099999

Modern societies are moving quickly towards digitalization. Beside software-based services, this paradigm is much dependent on mobile and embedded electronics, which in turn rely on enablers like advanced manufacturing and materials $[1,2]$. Curved, bendable and stretchable displays and other gimmicks are already entering the market. To be cost-effective in fabrication of electronic devices and materials for such applications, large-area and/or large-scale production is a necessity. Efficient tools such as roll-to-roll (R2R) manufacturing and quality monitoring are thus utilized [3, 4]. R2R production imitates methods used in paper and printing industries impressively: continuously rolling flexible base material (typically plastics) is step by step transformed into a functional product by adding features (conducts, wiring etc.) to it using printing, pressing, curing and similar techniques. As an essential part of the total quality assurance chain, testing during production ensures that the multistage processes are running as expected, and if not, gives feedback about quality issues as early as possible to locate sources of problems and to minimize yield losses.

Quality systems of electronics industry typically include measurement of several electrical and physical parameters as well as functional testing. Online or inline functional characterization of large area thin film electronics is challenging because of moving targets and diverse production environments. In addition, electrical testing techniques applied to traditional electronics production typically encounter problems in these applications, as they are not necessarily well compatible with large-sized, flexible materials. Previous studies have shown that optics based characterization by infrared (IR) imaging is very promising for this purpose [5-9]. Synchronized thermography (ST) has already proven its usefulness for continuous, online functional characterization of large area electronics [10]. Defects in indium tin oxide (ITO) on polyethylene terephthalate (PET) films were pointed out by the ST technique, and material uniformity and resistivity estimation of moving conductive thin film webs was realized on a R2R compatible environment online. On the previous setup, a galvanic contact was needed to electrically heat up the sample making vulnerable thin film structure exposed for mechanical stress. In addition, it was found to be impractical for samples with disconnected conductive structures. In order to overcome these problems, a fully contactless technique for characterization of moving conductive thin films using synchronized induction heating and thermal imaging is presented.

Induction heating is based on eddy current method, which is widely used in conductive material testing [11]. Eddy current inside the test sample is generated by placing the fluctuating magnetic field in proximity of it. This oscillating magnetic field is produced by exciting the conductive wire (coil) with alternating current. In contrast to the conventional eddy current testing (ECT), the impedance changes of the coil are not measured but induced current is used for electrical heating necessary for ST. The behavior of induced eddy current in the sample is influenced by the electrical properties of conductive thin film thus enabling the non-contact characterization of thin film. Earlier reports have shown that ECT can be utilized for non-destructive thin film electronics material testing [12-15]. However, due to the physical dimensions of the induction probe, a lateral resolution of this method is quite limited, and it is difficult to be implemented for large area samples. By combining eddy current heating with thermal imaging, this 
limitation of ETC method is bypassed thus helping to localize possible defects of large area thin films with good lateral resolution.

Analogous to earlier experiments [10], two continuous sample webs of conductive ITO on PET plastics were made out of material OC50 provided by Solutia - CPFilms Inc. The length of each continuous sample was set to $143 \mathrm{~cm}$ and the width to $15 \mathrm{~cm}$. One of the samples was handled carefully whereas another sample was mechanically stressed by bending and twisting. Thus, the stressed sample should have considerably more defects in the brittle ITO layer than the unstressed sample.

Similar R2R setup as previously was employed for experiments with an exception: this time, sample heating was generated by induction as shown on Fig. 1. An inducting heater was positioned just above $(<1 \mathrm{~cm})$ the sample film, and an IR camera pointed to an area right beside the heater. The width of the inductor was $20 \mathrm{~cm}$, so it reached diagonally over the sample web. An industrial grade induction system CELES MP25 made by Fives was utilized for heating. This heater has nominal power of $25 \mathrm{~kW}$. A value set to $4.5 \%$ on the power scale with frequency of $192 \mathrm{kHz}$ was practical to reach preferred temperature rise (below $10^{\circ} \mathrm{C}$ ) of the samples on the selected measurement location. Hollow aluminum-made heat shields were fixed around the tip of the induction heater, and air was purged through the shield beside the camera for cooling. Other main components of the setup (IR camera, rollers, etc.) remained as in Ref. [10]. The speed of the web was set to $2 \mathrm{~cm} / \mathrm{s}$ thus matching a value used in R2R production environments.

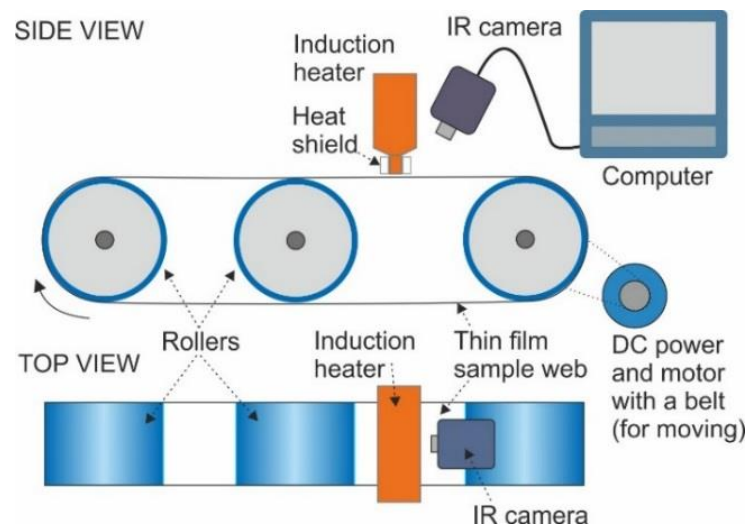

Fig. 1. Diagram of the R2R compatible test setup.

Compared with the work reported earlier [10], IR imaging and image pretreatment procedure was simplified because the recording of a reference temperature was left out, as it is not a necessity for further analysis and calculation of numerical quantities. Thus, this procedure was now performed as follows: 1) heating of the moving thin film web was set on; 2) a video sequence of one full round of the sample was stored to a temporary 3D image matrix; 3) a region of interest (ROI) of 1 x 330 pixels was selected at the same location on each frame of the temporary matrix and handed over to another matrix for compiling of a temperature map of a sample. A line-shaped ROI was applied to avoid issues with changes in emissivity caused by the angle of view. The height of the ROI corresponds to $89 \mathrm{~mm}$ on a sample, and so does the width of an IR image. The length of an image equals to the length of a sample belt so the actual size of captured image map covers an area of 1270 $\mathrm{cm}^{2}$. For estimation of uniformity, the same numerical quantity
$\Delta \mathrm{T}(\mathrm{x})$ introduced in Ref. [10] was used. The $\Delta \mathrm{T}(\mathrm{x})$ value shows transverse temperature deviation along an IR image map of a sample, and it is useful in pointing out defects in a sample thin film.

The IR images and $\Delta \mathrm{T}(\mathrm{x})$ plots of the samples are presented in Fig. 2. Both the thermograms and $\Delta \mathrm{T}(\mathrm{x})$ graphs reveal that the stressed ITO sample contains much more IR visible artifacts and shows much larger variation of $\Delta \mathrm{T}(\mathrm{x})$ than the non-stressed sample. The left (horizontal location $<10 \mathrm{~cm}$ ) and right (location $>130 \mathrm{~cm}$ ) edges of the thermograms are affected by the joint region of a continuous sample web, and they were left out of further analysis. As seen on Fig. 2 a), the stressed sample has tens of hot (yelloworange-red) spots whereas the non-stressed sample in Fig. 2 b) shows hardly any similar spots. These higher-temperature spots affect the $\Delta \mathrm{T}(\mathrm{x})$ plot, which expresses sharp peaks accordingly. Earlier experiments [7] specified that wide temperature variation is linked to defects in a thin film, so - as assumed - the stressed sample has considerably more defects than the unstressed sample. Beside sharp peaks, this kind of defected areas with higher and lower temperatures beside each other may also appear as gently sloping peaks, see e.g. horizontal location around $20 \mathrm{~cm}$ of Fig. 2 a). The stressed sample has a large defect on location $126 \mathrm{~cm}$ of Fig $2 \mathrm{a}$ ), which shows out features of both sharp and gently peaks. Defects found in the non-stressed were probably induced during installation of the sample to the test setup.

Table 1 presents calculated $\Delta \mathrm{T}(\mathrm{x})$ related values of the two ITOon-PET samples. Both the average of $\Delta \mathrm{T}(\mathrm{x})$ and its variation are notably larger for the stressed sample: the mean is about the double and the coefficient of variation is 21 percent units higher. Analysis of a $\Delta \mathrm{T}(\mathrm{x})$ limit reveals that much higher amount of the $\Delta \mathrm{T}(\mathrm{x})$ values stay above a certain limit for the stressed sample compared to the non-stressed sample. For example, when using a limit of $1.8^{\circ} \mathrm{C}$ almost half of the values for the stressed sample stay above the $\Delta \mathrm{T}(\mathrm{x})$ limit whereas less than $4 \%$ of the $\Delta \mathrm{T}(\mathrm{x})$ values for the unstressed sample exceed the same limit. This proposes that setting a $\Delta \mathrm{T}(\mathrm{x})$ limit and observing transcendences of this limit can thus be utilized for detecting likely defected regions of an ITO thin film web on-the-fly.

Table 1. $\Delta T(x)$ related values for the measurements of the samples shown in Fig. 2 (data between $10 \mathrm{~cm}$ to $130 \mathrm{~cm}$ ).

\begin{tabular}{lcc}
\hline & stressed & non-stressed \\
\hline mean of $\Delta \mathrm{T}(\mathrm{x})\left[{ }^{\circ} \mathrm{C}\right]$ & 2.069 & 1.130 \\
stdev of $\Delta \mathrm{T}(\mathrm{x})\left[{ }^{\circ} \mathrm{C}\right]$ & 1.009 & 0.316 \\
coefficient of variation $[\%]$ & 49.0 & 28.0 \\
over limit of $2.1^{\circ} \mathrm{C}[\%]$ & 29.4 & 0.5 \\
over limit of $1.8^{\circ} \mathrm{C}[\%]$ & 49.4 & 3.6 \\
over limit of $1.5^{\circ} \mathrm{C}[\%]$ & 80.3 & 13.9 \\
\hline
\end{tabular}

To get an indication about the consistency of the calculated $\Delta \mathrm{T}(\mathrm{x})$ values, three runs of each sample were conducted. The results of these tests tell that for the non-stressed sample, the mean $\Delta \mathrm{T}(\mathrm{x})$ stays on the level of $1.1^{\circ} \mathrm{C}$ with a minor $\left(<0.04^{\circ} \mathrm{C}\right)$ variation between runs, whereas for the stressed sample, the mean $\Delta \mathrm{T}(\mathrm{x})$ is about $2.2^{\circ} \mathrm{C}$ and the variation between runs is somewhat larger $\left(0.2^{\circ} \mathrm{C}\right)$. Our presumption is that the larger variation on the stressed sample is caused by the heating arrangement, such as irregular heating as for example the induction coil was not optimized for this kind of samples. Anyway, the mean of $\Delta \mathrm{T}(\mathrm{x})$ between runs retains well the same level for each sample indicating that this measurement technique is giving coherent and thus useful results. 


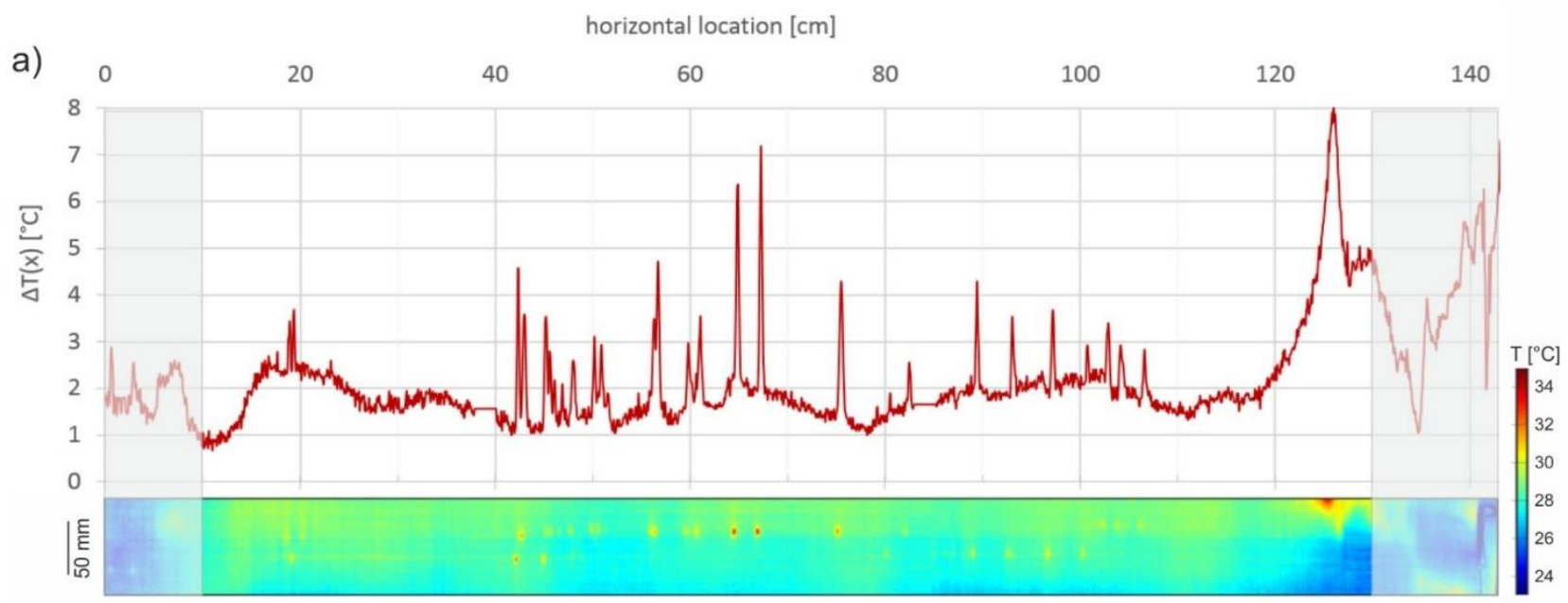

b)

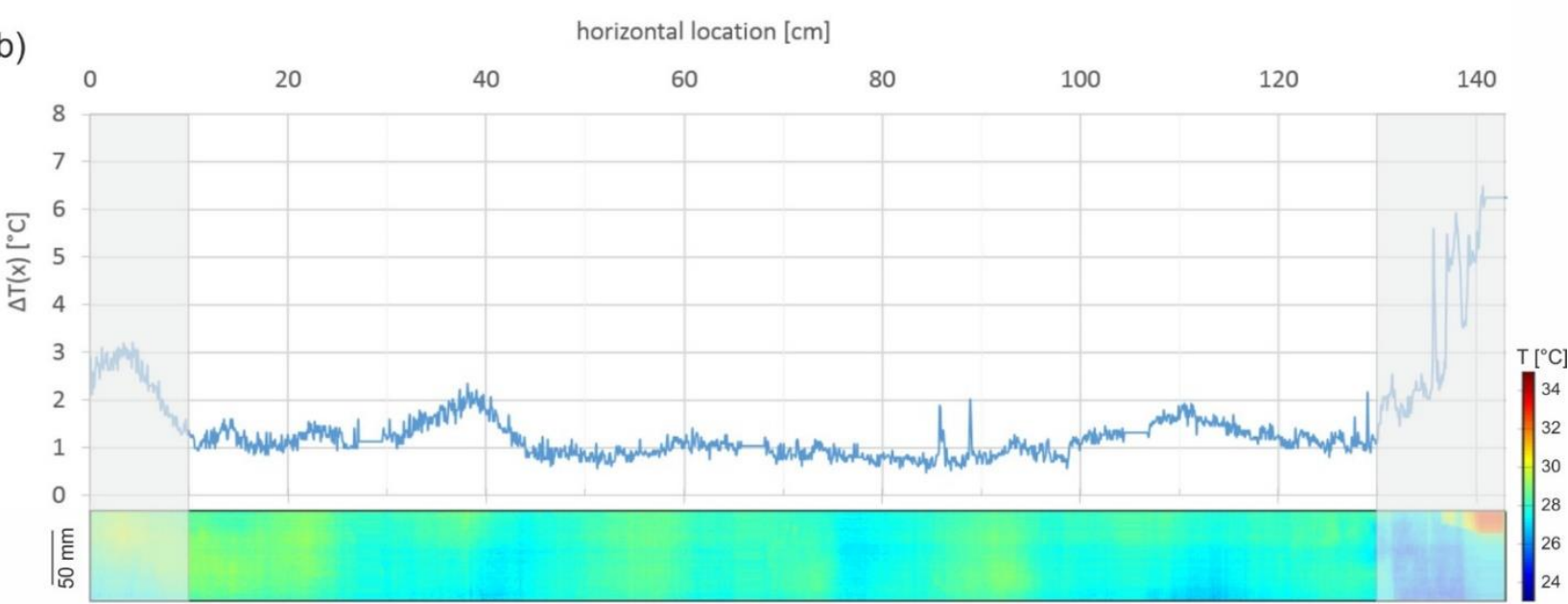

Fig. 2. Full-length IR image with corresponding $\Delta \mathrm{T}(\mathrm{x})$ plot of a) the stressed ITO on PET sample, and $\mathrm{b}$ ) the non-stressed ITO on PET sample. Areas marked with gray on both ends (horizontal location $<10 \mathrm{~cm}$ or $>130 \mathrm{~cm}$ ) were left out of analysis.

Earlier results [10] showed that a beneficial correlation between $\Delta \mathrm{T}(\mathrm{x})$ and resistance exists. To confirm that it is true also with the modified measurement technique, several resistance measurements with the well-known 4-wire technique were made along both samples using an Agilent 34401A multimeter and two probes (with two separate contacts each) having a distance of 50 $\mathrm{mm}$. This gap between probes was used because of an existing fixed-distance probe arrangement. Resistance was measured three times on 23 locations in the middle of the IR imaged areas shown in Fig. 2, and an average resistance for each sample was calculated using these measured values. The results of the conducted resistance measurements combined with the corresponding $\Delta \mathrm{T}(\mathrm{x})$ values are shown in Fig. 3, which reveals that a positive correlation between the measured resistance and the mean of $\Delta \mathrm{T}(\mathrm{x})$ values holds still true when using the measurement method presented in this paper. For the stressed sample, both the resistance $(62 \Omega$ vs 42 $\Omega$ ) and the mean $\Delta \mathrm{T}(\mathrm{x})\left(1.32^{\circ} \mathrm{C}\right.$ vs $\left.0.74^{\circ} \mathrm{C}\right)$ are evidently higher than for the non-stressed sample. Though this experiment may not prove the correlation comprehensively, it is still a strong indication of its existence. Thus, mean $\Delta \mathrm{T}(\mathrm{x})$ values can be utilized for estimating changes in electrical resistivity of thin films such as unexpected conductivity deterioration because of fabrication issues or problems in a conducting material itself.

As was pointed out, the technique proposed in this paper is able to locate defects, to estimate uniformity and to detect changes in electrical conductivity on moving, large-area conductive indium tin oxide thin films. These abilities are essential in functional and manufacturing perspectives as they enable online, real-time inspection of material and process issues. A characterizing method using contactless induction heating has notable advantages over a one requiring physical contact to the sample. Fully contactless inspection is certainly preferable because of flexibility and simplicity. Induction heating is far more versatile as it can placed in any section of a roll-to-roll production line that has some free space available. Thus, quality inspection of conductive thin films on multistage production may become feasible: measurements can be performed after each fabrication phase using fixedly installed thermal imaging and induction heating systems. Even ad hoc investigation of sudden quality issues becomes a reality with a portable type characterization system especially when no changes 
of a fabrication line itself are necessary - as is typically the case with techniques that need adding of some mechanical parts such as rolling metal-made disks. Without need for mechanical contacts, also the risk of damaging processed, often fragile materials is minimized.

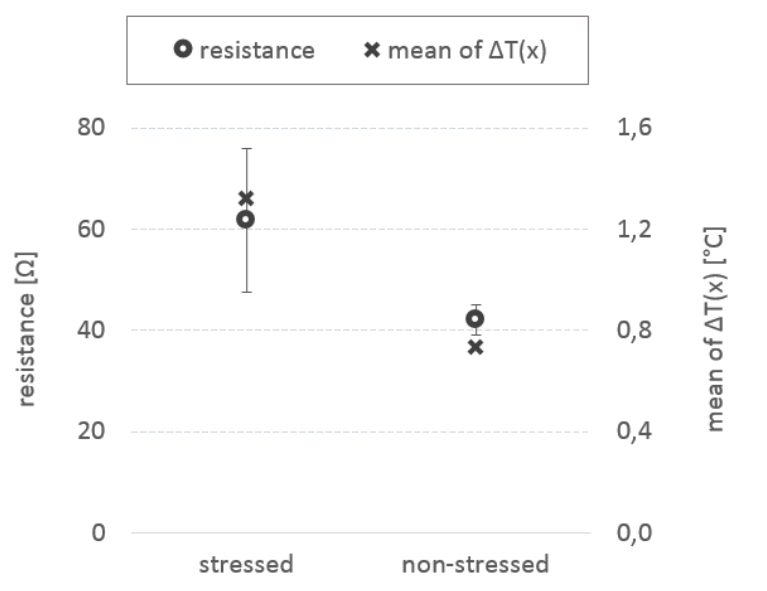

Fig. 3. Mean of $\Delta \mathrm{T}(\mathrm{x})$ and resistance values for the samples (data between $10 \mathrm{~cm}$ to $130 \mathrm{~cm}$ in lengthwise direction, $5 \mathrm{~cm}$ wide area in the middle of a sample). Deviation of the resistance is shown as bars.

Besides changing to a superior heating method, the original technique presented in [10] was streamlined otherwise, too. A reference temperature is not really needed for a technique like this one using temperature differences within a sample. Thus, already straightforward IR imaging process was simplified by leaving out recording of the reference temperature. Therefore, the calculation process of $\Delta \mathrm{T}(\mathrm{x})$ could also be eased making the technique even more well-matched for online/inline applications.

During the tests presented here, a quite bulky inductor head having the height of $18 \mathrm{~cm}$ was utilized. Considerably flatter and smaller inductors exist making it easier to incorporate them into an existing R2R system. The size and appearance of the inductor also affected the selection of measurement geometry. Here IR imaging was performed right after the heating step so that both phases were conducted from the same side (above) of the sample web. Because of limitations in placing the inductor, it was for example impossible to set up the inductor and the camera on opposite sides of the sample. Current measurement geometry was found workable, but further research is needed to confirm the usability of other interesting geometries.

In this paper, a constant frequency of induction heating was used which works well for a single layer thin film structure. Induced eddy current attenuates with depth in a sample depending on the conductivity and magnetic permeability of the tested material as well as the applied frequency [11]. Multi-frequency eddy current imaging has been applied e.g. to silicon solar cells, and it enables detection of issues inside multilayer structures [16]. Varying the frequency of inductive heating, it is thus expected that more complex R2R fabricated multilayer structures can be characterized with this technique as well, opening new possibilities for large area thin film electronics testing online.

Both the camera and the induction heater specify how wide area of the sample there is possible to inspect reasonably. The resolution, focal length and frame rate of the camera as well as the width of the heater are the main limiting factors. All those combined with the speed of the moving sample web affect the imaging performance. In this study, an IR camera equipped with a fixed focal length lens and an inductor covering the whole sample width were utilized. Performance and properties of the inductor and the camera were matching with the running speed of the web to produce usable images of the samples. Depending on the case, multi-camera/multiheater systems might be necessary, e.g. for wide samples, and a high-speed IR camera might be needed when the web moves quickly enough.

As a conclusion, an already capable online measurement technique was significantly enhanced by selecting a far more versatile heating method as well as simplifying the imaging and calculation procedures. The novel technique turned out to be even more useful as the original method by giving more degrees of freedom because of the touch-free, non-galvanic heating phase. IR imaging synchronized with induction heating appears as a highly promising tool for online quality control of large-area conductive thin films as was shown in the case of ITO on PET. The competent technique presented here should be usable also for other conducting materials and more complex structures used in printable electronics production thus giving it wide application potential.

Funding. Academy of Finland, Finnish Research Infrastructure 2018 (FIRI 2018) (320017).

Acknowledgment. Authors thank Mika Puirava from the Future Manufacturing Technologies group of the Kerttu Saalasti Institute for operating the induction heating equipment.

\section{References}

1. H. Kagermann, W. Wahlster, and J. Helbig, https://www.acatech.de/wpcontent/uploads/2018/03/ Final_report_Industrie_4.0_accessible.pdf.

2. D. Tokody, Procedia Manuf. 22, 1015 (2018).

3. J. Greener, G. Pearson, and M. Cakmak, Roll-to-Roll Manufacturing: Process Elements and Recent Advances (Wiley, 2018).

4. K. Seshan, Handbook of Thin Film Deposition: Techniques, Processes, and Technologies (Elsevier, 2012).

5. R. Ebner, B. Kubicek, G. Újvári, S. Novalin, M. Rennhofer, and M. Halwachs, Int. J. Photoenergy 2015, (2015).

6. A. Vetter, F.S. Babbe, B. Hofbeck, P. Kubis, M. Richter, S.J. Heise, J. Ohland, I. Riedel, and C.J. Brabec, Prog. Photovolt: Res. Appl. 24, 1001 (2016).

7. K. Leppänen, J. Saarela, R. Myllylä, and T. Fabritius, Opt. Express 21, 32358 (2013).

8. C. Schuss, K. Remes, K. Leppänen, J. Saarela, T. Fabritius, B. Eichberger, and T. Rahkonen, IEEE Trans. Instrum. Meas. 67, 1178 (2018).

9. B. Augustine, K. Remes, G. Lorite, J. Varghese, and T. Fabritius, Sol. Energy Mater. Sol. Cells 194, 74 (2019).

10. K. Remes, K. Leppänen, and T. Fabritius, Opt. Express 26, 2119 (2018).

11. X. Ma and A.J. Peyton, IEEE Trans. Instrum. Meas. 55, 570 (2006).

12. A. Fessant, J. Gieraltowski, J. Loaec, H. Le Gall, and A. Rakii, IEEE Trans. Magn. 29, 82 (1993).

13. E. van de Riet and F. Roozeboom, J. Appl. Phys. 81, 350 (1997).

14. V.J. Coates, Patent US4849694A (1989).

15. K. Chen, S. Nakamura, and A. Minamitsu, Patent US6700370B2 (2004).

16. H. Heuer, B. Hwang, M. Klein, M. Schulze, S. Hillmann, and N. Meyendorf, presented at the18th World Conference on Non-destructive Testing, Durban, South Africa, 16-20 April 2012. 